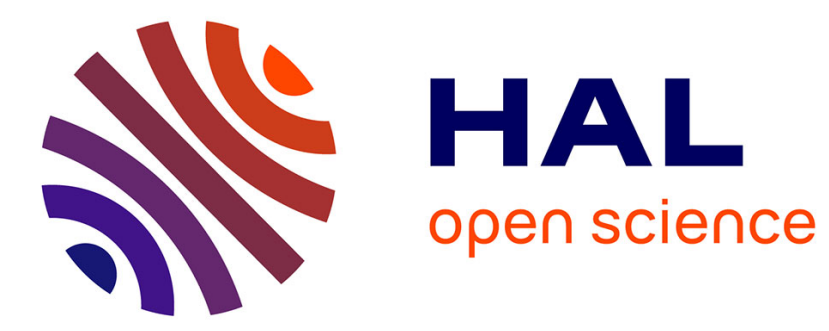

\title{
Use of Late-Life Expectancy for Assessing the Long-Term Benefit of Immune Checkpoint Inhibitors
}

\author{
Alexandre Vivot, Perrine Créquit, Raphaël Porcher
}

\section{To cite this version:}

Alexandre Vivot, Perrine Créquit, Raphaël Porcher. Use of Late-Life Expectancy for Assessing the Long-Term Benefit of Immune Checkpoint Inhibitors. JNCI: Journal of the National Cancer Institute, 2019, 111 (5), pp.519-521. 10.1093/jnci/djy211 . hal-02268387

\section{HAL Id: hal-02268387 \\ https://hal.science/hal-02268387}

Submitted on 20 Aug 2019

HAL is a multi-disciplinary open access archive for the deposit and dissemination of scientific research documents, whether they are published or not. The documents may come from teaching and research institutions in France or abroad, or from public or private research centers.
L'archive ouverte pluridisciplinaire HAL, est destinée au dépôt et à la diffusion de documents scientifiques de niveau recherche, publiés ou non, émanant des établissements d'enseignement et de recherche français ou étrangers, des laboratoires publics ou privés. 
This is a pre-copyedited, author-produced version of an article accepted for publication in JNCI: Journal of the National Cancer Institute following peer review. The version of record Vivot A, Créquit P, Porcher R. Use of Late-Life Expectancy for Assessing the Long-Term Benefit of Immune Checkpoint Inhibitors. J Natl Cancer Inst. 1 May 2019;111(5):519-21. is available online at: https://doi.org/10.1093/jnci/djy211 
Use of Late-life Expectancy for Assessing the Long-Term Benefit of Immune Checkpoint

\section{Inhibitors}

Short Running title: Long-term benefit of immune checkpoint inhibitors

Alexandre Vivot ${ }^{1,2}$, Perrine Créquit ${ }^{1,2} \&$ Raphaël Porcher $^{1,2}$

1. INSERM, UMR1153 Epidemiology and Statistics Sorbonne Paris Cité Research Center

(CRESS), METHODS Team, Paris, F-75004, France; Paris Descartes University, France.

2. Assistance Publique des Hôpitaux de Paris (AP-HP), Hôpital Hôtel Dieu, Centre

d'Épidémiologie Clinique, Paris, France.

Address correspondence to: Dr Alexandre Vivot. Centre d'épidémiologie clinique. Hôtel-Dieu de Paris. 1 place du parvis Notre-Dame. 75181 Paris cedex 04. France.

Tel: +331423478 12 Fax: +33142348790

email: alexandre.vivot@aphp.fr

Word count: 1000 / 1000 words

2 Figures

1 Supplementary Figure \& 1 Supplementary Table 


\begin{abstract}
To grade the long-term benefit of anticancer agents, the American Society of Clinical Oncology Value Framework (ASCO-VF) awards tail-of-the-curve bonus points by using milestone survival at twice the median control survival. Here we propose an alternative, late-life expectancy that we defined as the area under the Kaplan-Meier curve from median control survival to the end of follow-up. We analyzed all indications of immune checkpoint inhibitors with survival data and found that nine indications out of $13(69 \%)$ qualified for ASCO-VF tail of the curve bonus points either in progression-free or overall survival. Our proposed score recognized a long-term benefit not captured by the ASCO-VF, for example, for CHECKMATE66 where twice the median overall survival was not reached. We found that nivolumab was associated with an increase of 65\% (95\% CI: 39-90) in OS late-life expectancy, which highlights its important long-term benefit. In conclusion, the ASCO-VF could be improved with the use of late-life expectancy.
\end{abstract}

Key Words: Relative Value Scales; Prescription Drugs; Melanoma; Non-small Cell Lung Cancer; Survival Analysis; Immunotherapy 
Immune checkpoint inhibitors (ICIs) have raised hopes for durable survival. ${ }^{1,2}$ For assessing the value of anti-cancer drugs, the American Society of Clinical Oncology Value Framework (ASCO-VF) recognizes the long-term benefit by awarding tail-of-the-curve bonus points using milestone survival at twice the control median survival. ${ }^{3}$ This approach may have some drawbacks. First, milestones only reflect one point of the survival curve. ${ }^{4}$ Second, the milestone is not always reached with a short follow-up. Finally, dichotomization represents a loss of information ${ }^{5}$ and some drugs could fall short of the cut-off. Alternative treatment effect measures that could better estimate the magnitude of a drug's clinical benefit include the ratio of life expectancy (or restricted mean survival times). ${ }^{6,7}$ To quantify the long-term benefit of ICIs, Horiguchi et al. recently suggested using the area under the Kaplan-Meir curve between two milestones. ${ }^{8}$ Here, building on their idea, we propose a measure to quantify the long-term benefit of anticancer agents, the late-life expectancy, which we defined as the area under the Kaplan-Meier curve from median survival time in the control arm to the end of the follow-up.

We included all FDA-approved ICI indications for treatment of solid tumors from March 2011 to June 11, 2018. We used data from published reports of pivotal trials submitted to the FDA and first classified results from each trial for tail-of-the-curve bonus points. In ASCO-VF ${ }^{3}$, tail-of-the-curve bonus points are awarded with an improvement of at least $50 \%$ in survival provided the survival in the control group was at least $20 \%$. Bonus points are 20 points for overall survival (OS) and 16 for progression-free survival (PFS). For indications, in an overview of recently FDA-approved cancer drugs, the ASCO-VF total score ranged from 3.4 to 66.5 points. ${ }^{10}$

We then assessed long-term survival by computing, for each treatment arm, the late-life expectancy (LLE) as defined above. We reconstructed individual patient data (IPD) from the 
digitalized curves ${ }^{11}$ and then computed LLE with the R surv2sampleComp package. ${ }^{12}$ Because the median survival and the end of follow-up are not constants, we used non-parametric bootstrap with 999 replicates and computed the confidence interval (CI) with the bias-corrected and accelerated bootstrap techniques. Finally, we computed the ratio of LLE between experimental and control arms. Figure 1 exemplifies these calculations for the Checkmate 057 trial. $^{13}$

Finally, we proposed a scoring frame based on the ratios of LLEs. This formula was created such that it gives 20 points for OS (16 points for PFS) for a ratio of LLEs > 1.5 (to mimic the $50 \%$ relative difference in milestone survival that the ASCO-VF uses) and 0 points for a ratio $\leq 1$ and a bonus proportional to the ratio between 1 and 1.5 (with $20 \%$ lower scores for PFS as in the ASCO-VF).

From 2011 to June 11, 2018 the FDA approved six ICIs (ipilimumab, pembrolizumab, nivolumab, avelumab, atezolizumab, and durvalumab) in 27 indications (Supplementary Table 1). Half of the indications $(14 / 27,52 \%)$ were based on trials not reporting OS or PFS. Because the ASCO-VF requires OS or PFS data, we analyzed data for 13 trials (plus the updated analysis for Checkmate-057). Four out of 13 indications (31\%) reached the threshold for long-term benefit by the ASCO-VF in PFS (Supplementary Figure 1) and six indications out of $12(50 \%)$ in OS (Figure 2). Overall, nine indications out of 13 (69\%) qualified for ASCO-VF tail of the curve bonus points either in PFS or OS. This finding is different from that of a previous study in which only 3 out of 10 indications were awarded bonus points. ${ }^{14}$ The difference could be explained by the fact that we use Kaplan-Meier curves to estimate milestone survival in contrast of the previous study that used the proportion of patients still at risk at a given time. ${ }^{15}$ 
LLEs ratios and derived scores are depicted in Figure 2 for OS and in Supplementary Figure 1 for PFS. LLEs ratios ranged from 1.18 (Checkmate-025) to 1.89 (MDX010-20) for OS and from 1.28 (Checkmate-214) to 3.35 (MDX010-20) for PFS. For two indications, median control OS was not reached. Maturity of survival data is indeed a crucial point. A sufficient follow-up is necessary to observe medians and because high censoring will result in unstable estimates. Indeed, analyses of the same trial at different updates could yield very different estimates of the drug benefit. However, there is also a need to grade the long-term benefit as early as possible (e.g. for reimbursement decision). In our proposal, we not only give a score but also the ratio of LLE itself with its confidence interval so that one can directly assess the certitude around the estimate. It would be of great interest if the medical community could define, for each indication, the time point beyond which the treatment benefit is likely to remain stable. When we observe median control survival but not twice the median, calculation with our proposal is still possible. In Checkmate 66, no bonus points were awarded in OS because twice the median survival was not reached, ${ }^{16}$ but our analysis found a ratio of LLEs of $1.65(95 \%$ CI: 1.39 to 1.90 ) and a score of 20 points, which highlight the important long-term benefit of nivolumab. However, 16 bonus points were awarded in PFS in the ASCO-VF and thus the difference is only of 4 points at the indication level.

We showed that the use of LLEs ratios may provide a fine-grained assessment of longterm benefit. However, we only proposed a scoring to be further discussed and improved by the oncology community. For instance, to reward more precise studies, statistical significance could be easily incorporated in our approach, by assigning a null score for indications without a significant result or by using the lower bounds of the $95 \%$ CI instead of the point estimate to reward more precise studies. Further improvements could also considerate incorporate absolute 
magnitude of long term benefit in addition to relative effect. Our approach requires reconstructed (or original) IPD and re-analysis of these data, which might be a limitation of our approach.

\section{Funding}

None specific.

\section{Acknowledgements}

We acknowledge the paid contribution of Ms. Laura Smales (BioMedEditing, Toronto, Canada) for copyediting.

\section{Notes}

Authors do not have any conflict of interest linked to the current work.

\section{REFERENCES}

1. Paggio JCD. Immunotherapy: Cancer immunotherapy and the value of cure. Nat Rev Clin Oncol. February 2018. doi:10.1038/nrclinonc.2018.27

2. Temel JS, Gainor JF, Sullivan RJ, Greer JA. Keeping Expectations in Check With Immune Checkpoint Inhibitors. J Clin Oncol. January 2018:JCO.2017.76.2146. doi:10.1200/JCO.2017.76.2146

3. Schnipper LE, Davidson NE, Wollins DS, et al. Updating the American Society of Clinical Oncology Value Framework: Revisions and Reflections in Response to Comments Received. J Clin Oncol. 2016;34(24):2925-2934. doi:10.1200/JCO.2016.68.2518

4. Hellmann MD, Kris MG, Rudin CM. Medians and Milestones in Describing the Path to Cancer Cures: Telling “Tails.” JAMA Oncol. 2016;2(2):167-168. doi:10.1001/jamaoncol.2015.4345

5. Harrell F, Slaughter J. Biostatistics for Biomedical Research. 2016. http://fharrell.com/doc/bbr.pdf. Accessed March 14, 2018. 
6. Uno H, Claggett B, Tian L, et al. Moving Beyond the Hazard Ratio in Quantifying the Between-Group Difference in Survival Analysis. J Clin Oncol. 2014;32(22):2380-2385. doi:10.1200/JCO.2014.55.2208

7. Dehbi H-M, Royston P, Hackshaw A. Life expectancy difference and life expectancy ratio: two measures of treatment effects in randomised trials with non-proportional hazards. BMJ. 2017;357:j2250. doi:10.1136/bmj.j2250

8. Horiguchi M, Tian L, Uno H, et al. Quantification of Long-term Survival Benefit in a Comparative Oncology Clinical Study. JAMA Oncol. May 2018. doi:10.1001/jamaoncol.2018.0518

9. Center for Drug Evaluation and Research. Drug Approvals and Databases - Drugs@FDA Data Files. http://www.fda.gov/Drugs/InformationOnDrugs/ucm079750.htm. Accessed February $18,2016$.

10. Vivot A, Jacot J, Zeitoun J-D, Ravaud P, Crequit P, Porcher R. Clinical Benefit, Price and Approval Characteristics of FDA-approved New Drugs for Treating Advanced Solid Cancer, 2000-2015. Ann Oncol. 2017;28(5):1111-1116. doi:10.1093/annonc/mdx053

11. Guyot P, Ades AE, Ouwens MJ, Welton NJ. Enhanced secondary analysis of survival data: reconstructing the data from published Kaplan-Meier survival curves. BMC Med Res Methodol. 2012;12(1):9. doi:10.1186/1471-2288-12-9

12. Tian L, Uno H, Horiguchi M. Surv2sampleComp: Inference for Model-Free BetweenGroup Parameters for Censored Survival Data.; 2017. https://CRAN.Rproject.org/package $=$ surv2sampleComp.

13. Borghaei H, Paz-Ares L, Horn L, et al. Nivolumab versus Docetaxel in Advanced Nonsquamous Non-Small-Cell Lung Cancer. N Engl J Med. 2015;373(17):1627-1639. doi:10.1056/NEJMoa1507643

14. Ben-Aharon O, Magnezi R, Leshno M, Goldstein DA. Association of Immunotherapy With Durable Survival as Defined by Value Frameworks for Cancer Care. JAMA Oncol. 2018;4(3):326-332. doi:10.1001/jamaoncol.2017.4445

15. Vivot A, Créquit P, Porcher R. Improving on Tail-of-the-Curve Evaluation With the American Society of Clinical Oncology Value Framework. JAMA Oncol. August 2018. doi:10.1001/jamaoncol.2018.3289

16. Robert C, Long GV, Brady B, et al. Nivolumab in previously untreated melanoma without BRAF mutation. $N$ Engl J Med. 2015;372(4):320-330. doi:10.1056/NEJMoa1412082 


\section{Figure Legends}

Figure 1. Illustration of Late-life Expectancy Computation Based on Data from the CHECKMATE-057 trial. HR, hazard ratio; OS, overall survival; RMST, restricted mean survival times

Figure 2. Association Between Ratios of Late-life Expectancy and Tail-of-the-curve Bonus Points Within The ASCO-VF for Overall Survival. Note: There are only 12 indications (13 analyses with the CHECKMATE-057 update) represented because there was no overall survival data for PACIFIC study (Durvalumab in maintenance therapy of NSCLC).

Abbreviations: NSCLC, non-small-cell lung cancer; RCC, renal cell carcinoma. 


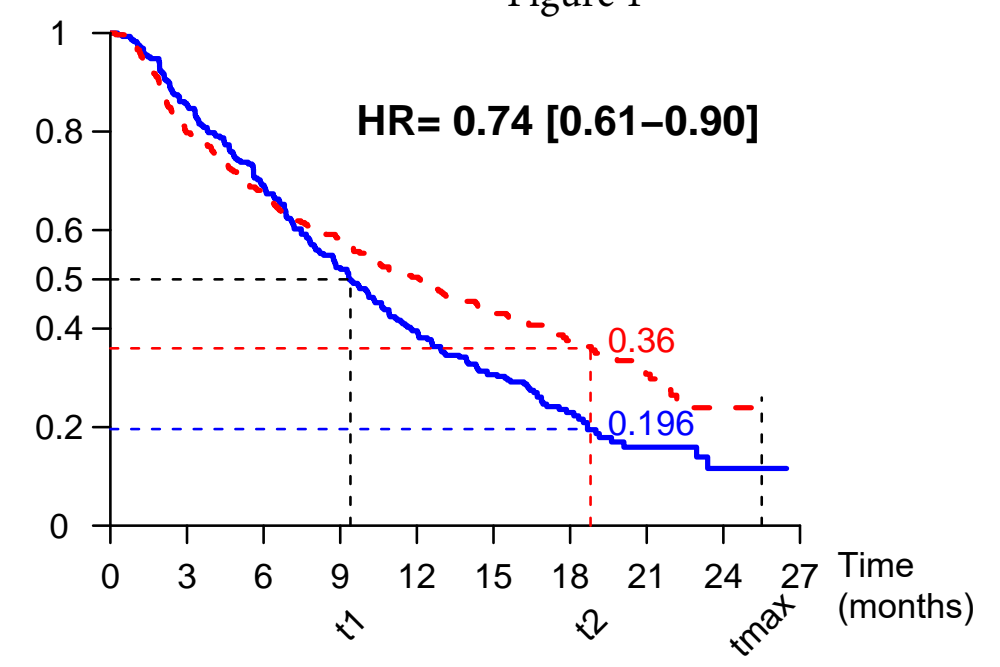

Figure 1

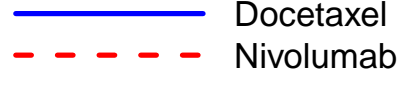

$\mathrm{t} 1$ : median survival time in the control arm

t2: twice $t 1$

tmax: end of follow-up

The relative difference in OS is $80 \%$.

However, this indication does not qualify for tail of the curve bonus points because survival at $\mathrm{t} 2$ is lower than $20 \%$.

OS $\quad$ RMST $=\mathbf{4 . 2}$ months

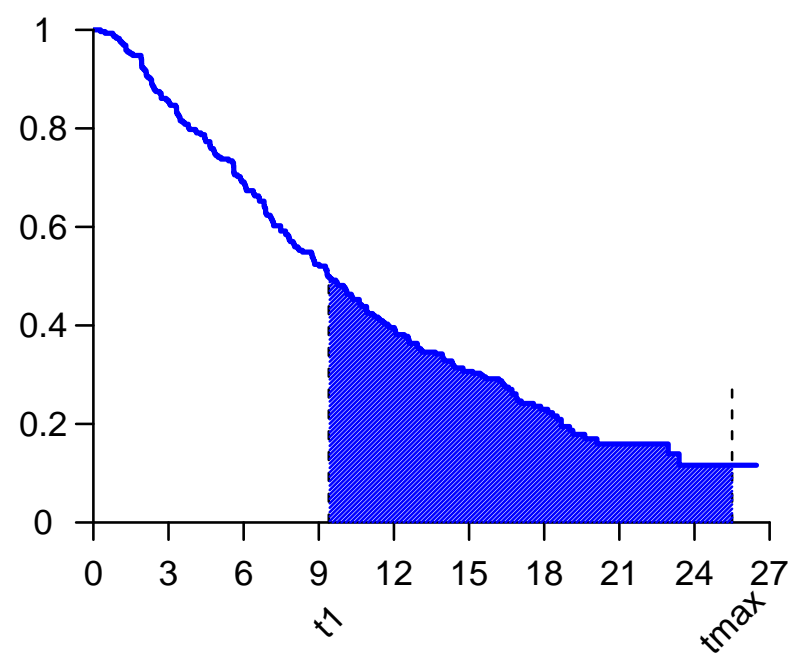

OS RMST $\mathbf{6} \mathbf{6}$ months

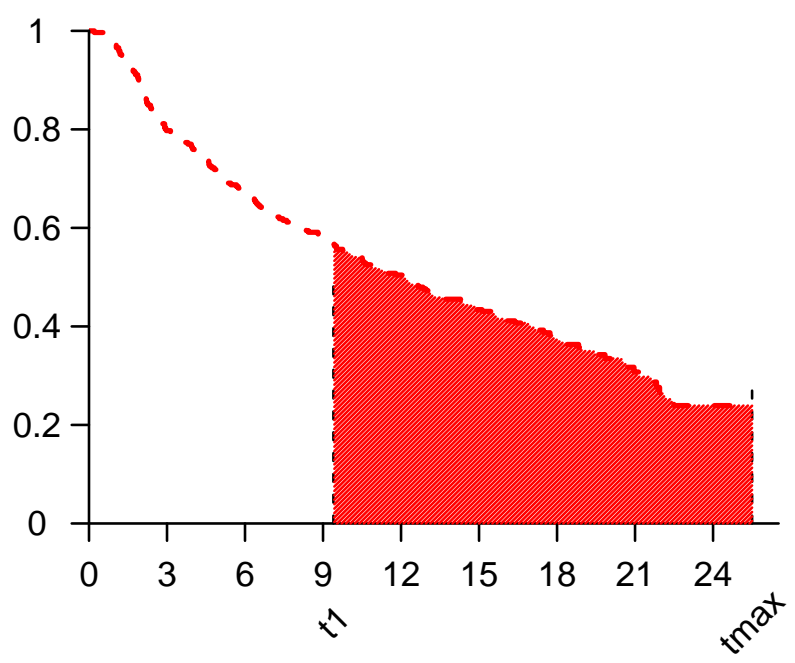

The ratio of RMST is 6.2 divided by 4.2 , i.e. 1.48 
Figure 2

\section{Drug, Indication}

\& Pivotal Trial
ASCO-VF Tail of the curve bonus points
Nivolumab NSCLC squamous

Second Line CHECKMATE-017

Nivolumab RCC Second Line CHECKMATE-025

No Bonus (T2 not reached)

Nivolumab NSCLC nonsquamous

Second Line CHECKMATE-057

No bonus

Nivolumab NSCLC nonsquamous Second Line CHECKMATE-057 update

Bonus

Nivolumab Melanoma

First Line CHECKMATE-066

No Bonus (T2 not reached)

Nivolumab HNSCC

Second Line CHECKMATE-141

Nivolumab + Ipilimumab RCC

First Line CHECKMATE-214

Pembrolizumab Melanoma

First Line KEYNOTE-006

Pembrolizumab NSCLC

Second Line KEYNOTE-010

Pembrolizumab NSCLC

First Line KEYNOTE-024

Pembrolizumab Urothelial carcinoma Second Line KEYNOTE-045

Ipilimumab Melanoma

Second Line MDX010-20

Atezolizumab NSCLC Second Line OAK

Bonus

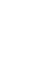

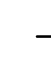

!

Median not reached

Bonus

Median not reached

Bonus

Bonus

No bonus

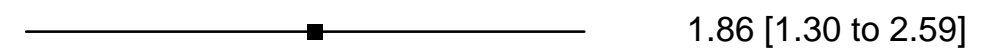

Ratio of Late Life Expectancy, months $[95 \% \mathrm{Cl}]$

1.86 [1.30 to 2.59$]$

$1.18[0.97$ to 1.40$]$

1.48 [1.19 to 1.80$]$

1.49 [1.19 to 1.82$]$

1.65 [1.39 to 1.90$]$

1.70 [1.31 to 2.29]

$1.21[0.97$ to 1.31$]$

$\mathrm{NA}$ [ NA to NA]

Proposed Bonus

$\mathrm{NA}$ [ NA to NA]

1.44 [1.15 to 1.76$]$

1.89 [1.24 to 2.72]

1.41 [1.18 to 1.68$]$

Ratio of Late Life Expextancy, months

$[95 \% \mathrm{Cl}]$ 
Online-only Table 1. Indications for Immune Checkpoint Inhibitors Approved by the US Food and Drug Administration (FDA) from March 2011 to May 2018.

\begin{tabular}{|c|c|c|c|c|c|c|}
\hline Drug & Indication & Line & $\begin{array}{l}\text { FDA approval } \\
\text { year }\end{array}$ & $\begin{array}{l}\text { Primary } \\
\text { endpoint }\end{array}$ & $\begin{array}{l}\text { RCT for FDA } \\
\text { approval }\end{array}$ & $\begin{array}{l}\text { Name of pivotal RCT } \\
\text { used to assess long- } \\
\text { term benefit }\end{array}$ \\
\hline Ipilimumab & Melanoma & First & 2015 & ORR & No & NA \\
\hline Pembrolizumab & Melanoma & Second & 2014 & ORR & No & NA \\
\hline Pembrolizumab & NSCLC & Second & 2015 & ORR & No & NA \\
\hline Pembrolizumab & HNSCC & Second & 2016 & ORR & No & NA \\
\hline Pembrolizumab & NSCLC & First & 2017 & ORR & No & NA \\
\hline Pembrolizumab & MSI-H & Second & 2017 & ORR & No & NA \\
\hline Nivolumab & Melanoma & Second & 2014 & ORR & No & NA \\
\hline Nivolumab & Urothelial carcinoma & Second & 2017 & ORR & No & NA \\
\hline Nivolumab & $\begin{array}{l}\text { Hepatocellular } \\
\text { Carcinoma }\end{array}$ & Second & 2017 & ORR & No & NA \\
\hline Nivolumab & MSI-H & Second & 2017 & ORR & No & NA \\
\hline Atezolizumab & Urothelial carcinoma & Second & 2016 & ORR & No & NA \\
\hline Avelumab & Merkel cell carcinoma & Second & 2017 & ORR & No & NA \\
\hline Avelumab & Urothelial carcinoma & Second & 2017 & ORR & No & NA \\
\hline
\end{tabular}




\begin{tabular}{|c|c|c|c|c|c|c|}
\hline Durvalumab & Urothelial carcinoma & Second & 2017 & ORR & No & NA \\
\hline Ipilimumab & Melanoma & Second & 2011 & OS & Yes & MDX010-20 \\
\hline Pembrolizumab & Melanoma & First & 2015 & OS & Yes & KEYNOTE-006 \\
\hline Pembrolizumab & Urothelial carcinoma & Second & 2017 & OS & Yes & KEYNOTE-045 \\
\hline Nivolumab & Melanoma & First & 2016 & OS & Yes & CHECKMATE-066 \\
\hline Nivolumab & Renal cell carcinoma & Second & 2015 & OS & Yes & CHECKMATE-025 \\
\hline Nivolumab & $\begin{array}{l}\text { Squamous-cell } \\
\text { NSCLC }\end{array}$ & Second & 2015 & OS & Yes & CHECKMATE-017 \\
\hline Nivolumab & $\begin{array}{l}\text { Non-squamous } \\
\text { NSCLC }\end{array}$ & Second & 2015 & $\mathrm{OS}$ & Yes & CHECKMATE-057 \\
\hline Nivolumab & HNSCC & Second & 2016 & OS & Yes & CHECKMATE-141 \\
\hline Atezolizumab & NSCLC & Second & 2016 & OS & Yes & OAK \\
\hline Pembrolizumab & NSCLC & First & 2016 & PFS & Yes & KEYNOTE-024 \\
\hline Durvalumab & NSCLC & Maintenance & 2018 & $\mathrm{PFS} / \mathrm{OS}$ & Yes & PACIFIC \\
\hline Pembrolizumab & NSCLC & Second & 2015 & PFS/OS & Yes & KEYNOTE-010 \\
\hline $\begin{array}{l}\text { Nivolumab + } \\
\text { ipilimumab }\end{array}$ & Renal cell carcinoma & First & 2018 & $\mathrm{PFS} / \mathrm{OS}$ & Yes & CHECKMATE-214 \\
\hline
\end{tabular}

RCT, randomized controlled trial; NSCLC, non-small-cell lung cancer; HNSCC, head and neck squamous cell carcinoma; MSI-H, high level microsatellite instability; OS, overall survival; PFS, progression-free survival; ORR, objective response rate; NA, not available 
Drug, Indication \& Pivotal Tria

ASCO-VF Tail of the Curve Bonus Points

Nivolumab NSCLC squamous Second Line CHECKMATE-017

Nivolumab RCC

Second Line CHECKMATE-025

Nivolumab NSCLC nonsquamous Second Line CHECKMATE-057

Nivolumab NSCLC nonsquamous Second Line CHECKMATE-057 update

Nivolumab Melanoma

First Line CHECKMATE-066

Nivolumab HNSCC

Second Line CHECKMATE-141

Nivolumab + Ipilimumab RCC

First Line CHECKMATE-214

Pembrolizumab Melanoma

First Line KEYNOTE-006

Pembrolizumab NSCLC Second Line KEYNOTE-010

Pembrolizumab NSCLC

First Line KEYNOTE-024

Pembrolizumab Urothelial carcinoma Second Line KEYNOTE-045

Ipilimumab Melanoma

Second Line MDX010-20

Atezolizumab NSCLC Second Line OAK

Durvalumab NSCLC Maintenance Line PACIFIC
Supplemental Figure 1. Association Between Ratios of Late-life Expectancy and Tail-of-thecurve Bonus Points Within The ASCO-VF for

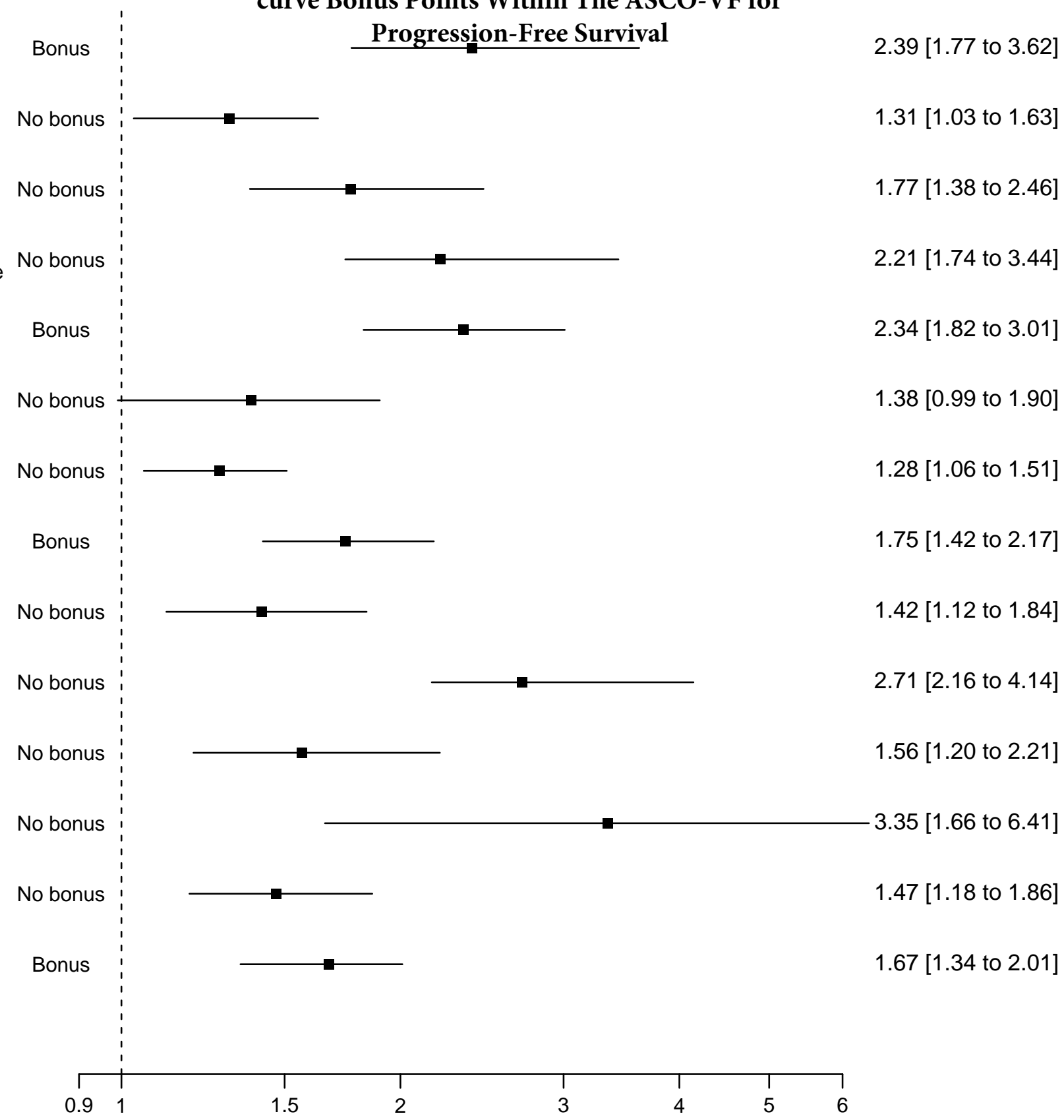

